\title{
THE COBORDISM OF INVOLUTIONS ON ORIENTABLE MANIFOLDS
}

\author{
R. J. ROWLETT
}

\begin{abstract}
This note calculates the cobordism of smooth, oriented manifolds with orientation-reversing involution, and also the 2-torsion in the cobordism of such manifolds with orientationpreserving involution. In particular, it is shown that the forgetful homomorphism maps both groups monomorphically into the cobordism of unoriented manifolds with involution.
\end{abstract}

1. Statement of results. Let $\mathcal{O}_{*}$ be the cobordism theory of smooth oriented manifolds $M$ with involution $T$; we do not require $T$ to be orientation preserving. Then $\mathcal{O}_{*}=\mathcal{O}_{*}^{-} \oplus \mathcal{O}_{*}^{+}$, where the equivalence class $[M, T]$ is in $\mathcal{O}_{*}^{-}$(respectively, $\mathcal{O}_{*}^{+}$), if $T$ is orientation reversing (respectively, preserving). The notation agrees with that of [4].

In this note we determine $\mathcal{O}_{*}^{-} \oplus$ torsion $\mathcal{O}_{*}^{+}$. Rosenzweig [5] proved that the torsion of $\mathcal{O}_{*}^{+}$is all of order 2 . In $\mathcal{O}_{*}^{-}$every class is of order 2 , for if $T$ is orientation reversing,

$$
(M \cup M, T \cup T) \cong \partial(M \times I, T \times \mathrm{Id})
$$

under the diffeomorphism Id $\cup T$.

$\mathcal{O}_{*}^{+}$was also studied by Conner [2], and $\mathcal{O}_{*}$ has recently been studied by Lee and Wasserman [4], who show how classes of $\mathcal{O}_{*}$ are detected by equivariant characteristic numbers. Our results complement these two papers, and we offer a short proof (Lemma 4) of a result which appears in both.

THEOREM 1. Let $I_{*}$ be the cobordism of unoriented manifolds with involution [3, Chapter IV]. The forgetful map $\rho: \mathcal{O}_{*} \rightarrow I_{*}$ is monic on 2torsion.

From [6] it follows that the torsion is mapped monomorphically into the Wall bordism group $\tilde{W}_{*}\left(\bigvee_{j=1}^{\infty} T B O(j)\right)$ (see [7, Chapter VIII]). An element of this group is represented by a vector bundle $\nu \rightarrow F$, whose disk bundle $D v$ is a Wall manifold [8], together with a classifying map

Received by the editors November 14, 1972.

AMS (MOS) subject classifications (1970). Primary 57D85; Secondary 57D85.

Key words and phrases. Equivariant cobordism, orientation-preserving involution, orientation-reversing involution, Wall manifold.

(c) American Mathematical Society 1973 
$(D v, S v) \rightarrow(\bigvee T B O(j), \infty)$. Define $\partial^{\prime}[D v]=\left[D i^{*} v\right]$, where $i: N \subset F$ includes a submanifold dual to $w_{1} F+w_{1} v$. Then $D i^{*} v$ is dual to $w_{1} D v$, hence is oriented.

THEOREM 2. $\mathcal{O}_{*}^{-} \cong \operatorname{Im} \partial^{\prime} \cap \tilde{W}_{*}\left(\bigvee_{j=1}^{\infty} T B O(2 j+1)\right)$.

Let $p: \tilde{W}_{*}(\bigvee T B O(2 j)) \rightarrow W_{*}$ map $[D v]$ to the class of the projective space bundle $P v$, and $q: \tilde{W}_{*}(T B O(1)) \rightarrow \tilde{W}_{*}(\bigvee T B O(2 j))$ map [Dv] to $[D(v \oplus 1)]$. Then $p q=0$ and $\partial^{\prime}(\operatorname{Im} q) \subseteq \operatorname{Im} q$, so $p$ and $\partial^{\prime}$ induce

and

$$
p^{\prime}: \tilde{W}_{*}(\bigvee T B O(2 j)) / \operatorname{Im} q \rightarrow W_{*}
$$

$$
\partial^{\prime \prime}: \tilde{W}_{*}(\bigvee T B O(2 j)) / \operatorname{Im} q \rightarrow \tilde{W}_{*}(\bigvee T B O(2 j)) / \operatorname{Im} q .
$$

THEOREM 3. torsion $\mathcal{O}_{*}^{+} \cong \operatorname{Im} \partial^{\prime \prime} \cap \operatorname{Ker} p^{\prime}$.

These theorems are used to describe $Z_{2}$-generators of $\mathcal{O}_{*}^{-}$and torsion $\mathcal{O}_{*}^{+}$. The usefulness of the latter description is limited by lack of knowledge of $\operatorname{Ker} p^{\prime}$.

I wish to thank Professors Lee and Wasserman for supplying an advance copy of [4], and also Professor R. E. Stong for his advice and encouragement.

2. Wall manifolds with involution. A Wall manifold with involution, $(W, T, f)$, is an unoriented manifold $W$ with an involution $T$ and a map $f: W \rightarrow S^{1}$ such that $f T=f$ and $f^{*}(\iota)=w_{1} W$, where $\iota \in H^{1}\left(S^{1} ; Z_{2}\right)$ is the generator. To construct examples one uses:

Lemma 1 (See [6, (2.5)]). If $D v$ is a Wall manifold (respectively, orientable) and $\operatorname{dim} v$ is even, then $P \nu$ is a Wall manifold (respectively, orientable).

Let $W_{*}^{I}$ and $W_{*}^{F^{\prime}}$ be the cobordism groups of Wall manifolds with involution and with fixed point free involution, respectively. Following are the chief results of [6]. The forgetful map $W_{*}^{I} \rightarrow I_{*}$ is monic. Clearly $\operatorname{Im} \rho \subseteq W_{*}^{I}$. If $d: W_{*}^{I} \rightarrow \mathcal{O}_{*}$ assigns to $[W, T, f]$ the class of $(N, T \mid N)$, where $N \subset W$ is an invariant submanifold dual to $w_{1} W$, then the sequence

$$
\mathcal{O}_{*} \stackrel{\rho}{\longrightarrow} W_{*}^{I} \stackrel{d}{\longrightarrow} \mathcal{O}_{*} \stackrel{2}{\longrightarrow} \mathcal{O}_{*}
$$

is exact. Define $d^{\prime}=\rho d: W_{*}^{I} \rightarrow W_{*}^{I}$. The map $s: W_{*}^{I} \rightarrow \tilde{W}_{*}(\bigvee T B O(j))$, given by classifying the normal bundle to the fixed set, is monic. The map $\partial: \tilde{W}_{*}(\bigvee T B O(j)) \rightarrow W_{*}^{F}$, given by $\partial[D v]=[S v$, antipodal map $]$, is epic.

We give a new proof of the last fact by constructing a splitting map. Recalling [6, (3.1)], if $[M, f] \in W_{*}\left(R P^{\infty}\right)$ let $\pi: \tilde{M} \rightarrow M$ be the double cover classified by $f$. If $[M, f] \in N_{*}\left(S^{1}\right)$ let $\pi: \tilde{M} \rightarrow M$ be the double cover corresponding to $f^{*} \iota+w_{1} M$. In either case $\tilde{M}$ is a Wall manifold with 
involution $\Delta$, the deck transformation reversing sheets of $\tilde{M}$. This defines monomorphisms

$$
k: W_{*}\left(R P^{\infty}\right) \rightarrow W_{*}^{F}, \quad j: N_{*}\left(S^{1}\right) \rightarrow W_{*}^{F}
$$

with $k+j$ epic and $\operatorname{Im} k \cap \operatorname{Im} j \cong W_{*}$, identified as the set of classes $\left[W \times S^{0}, \operatorname{Id} \times \Delta\right]$ for $[W] \in W_{*}$.

Now let $\lambda \rightarrow M$ be the line bundle corresponding to $\pi$. If $[M, f] \in N_{*}\left(S^{1}\right)$, $D \lambda$ is a Wall manifold. Define

$$
\delta[\tilde{M}, \Delta]=[D \lambda] \in \tilde{W}_{*}(\bigvee T B O(n)) .
$$

If $[M, f] \in W_{*}\left(R P^{\infty}\right)$, let $i: N \subset M$ include a submanifold defining the double cover. $D\left(i^{*} \lambda\right)$ is a Wall manifold; by Lemma 1 so is $P=P\left(i^{*} \lambda \oplus 1\right)$. Define

$$
\delta[\tilde{M}, \Delta]=[D(i * \lambda \oplus 1)]+[D(1 \rightarrow M)] .
$$

LEMMA 2. $\partial \delta=\mathrm{Id}: W_{*}^{F} \rightarrow W_{*}^{F}$.

Proof. For $x \in \operatorname{Im} j$ it is obvious that $\partial \delta(x)=x$. If $[M, f] \in W_{*}\left(R P^{\infty}\right)$ write $P=D\left(i^{*} \lambda\right) \cup D\left(i^{*} \lambda\right)$. The map $g: P \rightarrow R P^{\infty}$, classifying the canonical line bundle, may be written

$$
g=(f \mid N) p_{0} \cup *
$$

where $p_{0}$ is the projection of $D\left(i^{*} \lambda\right)$ and $*$ is homotopic to a trivial map (see the proof of $[3,(26.1)]$ ). Since $i^{*} \lambda$ is the normal bundle of $N$ in $M$ we may also assume $f$ is of the form (1). Now form $(H, h)$ from $\left(M \times I, f \pi_{M}\right)$ and $\left(P \times I, g \pi_{P}\right)$ by sewing copies of $\left(D\left(i^{*} \lambda\right) \times 1,(f \mid N) p_{0}\right)$.

$\partial(H, h)=(M, f) \cup(P, g) \cup(M, *)$, which proves $\partial \delta[\tilde{M}, \Delta]=[\tilde{M}, \Delta]$.

3. Proof of Theorem 1. The statement of the theorem can be sharpened:

THEOREM $1^{\prime}$. $\rho$ maps torsion $\mathcal{O}_{*}$ isomorphically onto $\operatorname{Im} d^{\prime}$.

Lemma 3. Let $(M, T)$ be an orientable manifold with involution, and $(M, T)=\partial(Q, U)$ as unoriented manifolds with involution. If $Q$ is in fact orientable, then

$$
[M, T]=2 \beta+\omega\left[S^{0}, \Delta\right]
$$

for some $\beta \in \mathcal{O}_{*}^{+}$and $\omega \in \Omega_{*}$.

Proof. Give $Q$ an orientation and let $\partial Q=M^{\prime}$ be its oriented boundary.

$$
[M, T]=[M, T]-\left[M^{\prime}, T\right] .
$$

Of course $M^{\prime}=M$ up to the sign of the orientation on each component. $2 x=0$ for $x \in \mathcal{O}_{*}^{-}$; also the involution $M \cup(-M) \rightarrow M \cup(-M)$, reversing components, bounds $(M \times[-1,1], \operatorname{Id} \times(-1))$.

(2) then reduces to the desired form. 
LEMMA 4. If $x \in \mathcal{O}_{n}$ and $\rho x=0$ then either $x \in 2 \mathcal{O}_{n}$ or else $n=4 i$ and

$$
x=2 \beta+\omega\left[S^{0}, \Delta\right]
$$

for some $\beta \in \mathcal{O}_{4 i}^{+}$and $\omega \in \Omega_{4 i}$ torsion.

Note. This is [4, Proposition 21] and, essentially, [2, Theorem 5.8]. Following is a short proof relying on [6].

Proof. Write $x=y+z, y \in \mathcal{O}_{*}^{+}, z \in \mathcal{O}_{*}^{-}$. Since $s \rho y$ and $s \rho z$ lie in orthogonal summands we must have $\rho y=0=\rho z$.

Let $(M, T)$ represent either $y$ or $z$, and let $(W, S, f)$ be a Wall manifold with involution and boundary $(M, T, *)$. By $[6,(4.1)]$ we may assume $f(M)=\{a\}$ and $f$ is transverse regular at $b \neq a$. If $b \in \operatorname{Im} f$, cut $W$ along $N=f^{-1} b$, as in $[8, \S 1]$, obtaining an oriented cobordism of $(M, T)$ with $\left(N \times S^{0},(S \mid N) \times \operatorname{Id}\right)$. This bounds if $S$ is orientation reversing. If $b \notin \operatorname{Im} f$, then $W$ is orientable and Lemma 3 applies. Finally, Rosenzweig showed [5] that $2 \beta=0$ if $n \neq 4 i$ and that $\omega\left[S^{0}, \Delta\right]=0$ if $2 \omega=0$.

Suppose $x \in \mathcal{O}_{n}, \rho x=0=2 x$. If $x \in 2 \mathcal{O}_{n}$ then $x=0$, for the only torsion is of order 2. If $x$ has the form (3), then since $2 x=0$ we have $4 \beta=2 \omega\left[S^{0}, \Delta\right]$. By the proof of [2, Corollary 5.5], index $(\omega)$ is even. Then [2, Theorem 4.5] proves $\omega\left[S^{0}, \Delta\right] \in 2 \mathcal{O}_{4 i}^{+}$. Thus $x=0$ in this case also, and $\rho$ is monic on the torsion. The reader can easily show that $\rho$ (torsion) $=\operatorname{Im} d^{\prime}$, completing Theorem 1'.

4. Calculation of $\partial^{\prime}$. To give meaning to Theorems 2 and 3 , we compute the homomorphism $\partial^{\prime}$. The structure of $\tilde{W}_{*}(\bigvee T B O(n))$ is determined by $[3, \S 17]$ and $[7$, pp. 163-164].

LemMa 5. $\tilde{W}_{*}(\bigvee T B O(n))$ is the $W_{*}$-polynomial algebra on generators $\left[D \lambda_{i}\right]$, one of each dimension $i \geqq 1$, with $\lambda_{i}$ a line bundle. If $i=2 m+1$, we may take $\lambda_{i}$ to be the canonical line bundle over $R P^{2 m}$. If $i=2 m+2$, we may take $\lambda_{i}$ to be the canonical line bundle over $P\left(\xi \oplus 2 m \rightarrow S^{1}\right)$, where $\xi \rightarrow S^{1}$ is the nontrivial line bundle.

The proof is perfectly straightforward, and all the needed cohomology data is listed in $[6,(4.1)]$.

Recall that $\partial^{\prime}[D v]=\left[D i^{*} v\right]$, if $\nu \rightarrow F$ and $i: N \subset F$ includes a submanifold dual to $w_{1} v+w_{1} F$. By [7, pp. 169-172], $D v$ is orientable if and only if $[D v] \in \operatorname{Im} \partial^{\prime}$. For future reference, if $v$ has even fiber dimension then by [6, (2.4)] it is also true that $P\left(i^{*} v\right)$ is dual in $P \nu$ to $w_{1} P \nu$. Then

by [8].

$$
2\left[P\left(i^{*} v\right)\right]=0 \in \Omega_{*},
$$


LEMMA 6. For all $m, \partial^{\prime}\left[D \lambda_{2 m+2}\right]=\left[D \lambda_{2 m+1}\right]$ and $\partial^{\prime}\left[D \lambda_{2 m+1}\right]=0$. If $\partial_{1}: W_{*} \rightarrow W_{*}$ maps $W$ to the submanifold dual to $w_{1} W[8]$, then

$$
\partial^{\prime}(w \beta)=\left(\partial_{1} w\right) \beta+w^{\prime}\left(\partial^{\prime} \beta\right)
$$

for all $w \in W_{*}, \beta \in \tilde{W}_{*}(\bigvee T B O(n))$.

REMARKS. The first statement is immediate and the second may be proved with characteristic numbers, exactly like [8, Lemma 3]. From the proof of $[6,(4.1)]$, we see that $\partial^{\prime} s=s d^{\prime}$. Since $s$ is a $W_{*}$-monomorphism, the lemma also proves that $d^{\prime}(w \beta)=\left(\partial_{1} w\right) \beta+w^{\prime}\left(d^{\prime} \beta\right)$ for $w \in W_{*}, \beta \in W_{*}^{I}$.

5. Proof of Theorems 2 and 3. If $v$ has odd fiber dimension, then by $[6,(3.3)]$ we have $\delta \partial[D v]=[D \lambda]$, where $\lambda \rightarrow P \nu$ is the canonical line bundle. By Lemma $1, P(v \oplus 1)$ is a Wall manifold. Let $t(v)$ be the involution on $P(\nu \oplus 1)$ induced by the linear map $1 \times(-1)$ in each fiber of $\nu \oplus 1$, and define

$$
\sigma[D v]=[P(v \oplus 1), t(v)] \in W_{*}^{I} .
$$

Then $s \sigma[D v]+\delta \partial[D \nu]=[D \nu]$, just as in $[3, \S 28]$. It follows that $\sigma[D v]=0$ if and only if $[D \nu]=\delta \partial[D \nu] \in \tilde{W}_{*}(T B O(1))$. Also, since $\partial s=0$, it follows that $\sigma s \rho(x)=\rho(x)$ for $x \in \mathcal{O}_{*}^{-}$. Since $P(\nu \oplus 1)$ is orientable if $D v$ is, and $t(v)$ is then orientation reversing, Theorem 2 is proved.

If $\nu \rightarrow F$ has even fiber dimension then we will assume $[D \nu] \in \operatorname{Ker} p$. To justify this restriction, suppose $[M, T] \in \mathcal{O}_{*}^{+}$and $v$ is the normal bundle to the fixed set of $T$. Then $P v$ bounds $(M-\operatorname{Int} D v) / T$, so $p s \rho[M, T]=0$.

Suppose, then, that $[D v] \in \operatorname{Ker} p$. Since $P v$ is a Wall manifold, Lemma 2 implies $\delta[D v]=\left[D\left(i^{*} \lambda \oplus 1\right)\right]$, where $i: N \subset P \nu$ includes a submanifold dual to $\lambda$. We define $\sigma: \operatorname{Ker} p \rightarrow W_{*}^{I}$. Let $\tilde{N}=S\left(i^{*} \lambda\right)$, so $\tilde{N} \rightarrow N$ is a double cover and $\tilde{N}$ is imbedded in $S v$ with trivial normal bundle. $\tilde{N}$ is also imbedded in $S\left(i^{*} \lambda \oplus 1\right)$ with trivial normal bundle, so we sew $D v$ and $D(i * \lambda \oplus 1)$ along the copies of $\tilde{N} \times I$ in the boundaries. The remaining boundary is then two copies of $P v$, which bounds some $Q$. Sewing on $2 Q$ builds a Wall manifold $K(v)$, which admits an involution $t(v)$ that restricts to the antipodal involution on the disk bundles, and reverses the two copies of $Q$. Thus the fixed set of $t(v)$ is the disjoint union of $F$ and $N$, and the normal bundles are $\nu$ and $i * \lambda \oplus 1$, respectively.

Define $\sigma[D v]=[K(v), t(v)] \in W_{*}^{I}$; it is easy to see that this is a welldefined homomorphism. By construction,

$$
s \sigma[D \nu]+\delta \partial[D \sigma]=[D \nu] .
$$

Reasoning as before, $\sigma[D \nu]=0$ if and only if $[D \nu]=\delta \partial[D \nu] \in \operatorname{Im} q$, and $\sigma s \rho(x)=\rho(x)$ for $x \in \mathcal{O}_{*}^{+}$. 
Finally, suppose $D v$ is oriented: Then so are $D\left(i^{*} \lambda \oplus 1\right)$ and $P \nu$. As noted in the last section, $P \nu$ represents a torsion class in $\Omega_{*}$. Since $\Omega_{*} \rightarrow W_{*}$ is monic on torsion [8] we may assume $Q$ is oriented. Thus $K(v)$ is oriented, and $t(v)$ is orientation preserving. This completes the proof of Theorem 3.

RemarKs. (1) We have shown that $\mathcal{O}_{*}^{-}$is the $Z_{2}$-vector space on generators $[P(\nu \oplus 1), t(v)]$, for $[D v] \in \operatorname{Im} \partial^{\prime}, \operatorname{dim} v=2 m+1, m>0$. The torsion of $\mathcal{O}_{*}^{+}$is a $Z_{2}$-vector space on generators $[K(v), t(v)]$ for

$$
[D v] \in \operatorname{Im} \partial^{\prime \prime} \cap \operatorname{Ker} p^{\prime},
$$

but this is not very helpful, since we cannot actually construct $K(v)$ without knowing $Q=\partial P \nu$.

(2) If $[M, T] \in \mathcal{O}_{*}^{-}$, then clearly $[M]$ is a torsion class in $\Omega_{*}$. Conversely, P. G. Anderson proved [1, Proposition 5] that every torsion class in $\Omega_{*}$ is represented by one of our examples $P(\nu \oplus 1)$.

\section{REFERENCES}

1. P. G. Anderson, Cobordism classes of squares of orientable manifolds, Ann. of Math. (2) 83 (1966), 47-53. MR 32 \#4700.

2. P. E. Conner, Orientation-preserving involutions, Lectures on the action of a finite group, Lecture Notes in Math., no. 73, Springer-Verlag, Berlin, 1968, Chap. 2. MR 41 \#2670.

3. P. E. Conner and E. E. Floyd, Differentiable periodic maps, Ergebnisse der Mathematik und ihrer Grenzgebiete, Heft 33, Academic Press, New York; SpringerVerlag, Berlin, 1964, MR 31 \#750.

4. C. N. Lee and A. G. Wasserman, Equivariant characteristic numbers, Proc. Second Conf. Transformation Groups, part I, Lecture Notes in Math., vol. 298, Springer-Verlag, Berlin, 1972, pp. 191-216. 1-10.

5. H. L. Rosenzweig, Bordism of involutions on manifolds, Illinois J. Math 16 (1972).,

6. R. J. Rowlett, Wall manifolds with involution, Trans. Amer. Math. Soc. 169 (1972), 153-162.

7. R. E. Stong, Notes on cobordism theory, Princeton Univ. Press, Princeton, N.J.; Univ. of Tokyo Press, Tokyo, 1968. MR 40 \#2108.

8. C. T. C. Wall, Determination of the cobordism ring, Ann. of Math. (2) 72 (1960), 292-311. MR 22 \#11403.

Department of Mathematics, Princeton University, Princeton, New Jersey 08540 